\title{
Second Order Regularity for a Linear Elliptic System Having BMO Coefficients
}

\author{
Gioconda Moscarielloø and Giulio Pascaleø
}

\begin{abstract}
We consider linear elliptic systems whose prototype is

$$
\operatorname{div} \Lambda[\exp (-|x|)-\log |x|] I D u=\operatorname{div} F+g \text { in } B .
$$

Here $B$ denotes the unit ball of $\mathbb{R}^{n}$, for $n>2$, centered in the origin, $I$ is the identity matrix, $F$ is a matrix in $W^{1,2}\left(B, \mathbb{R}^{n \times n}\right), g$ is a vector in $L^{2}\left(B, \mathbb{R}^{n}\right)$ and $\Lambda$ is a positive constant. Our result reads that the gradient of the solution $u \in$ $W_{0}^{1,2}\left(B, \mathbb{R}^{n}\right)$ to Dirichlet problem for system $(0.1)$ is weakly differentiable provided the constant $\Lambda$ is not large enough.
\end{abstract}

Mathematics Subject Classification. 35B65, 35J57, 47B47.

Keywords. Linear elliptic systems, BMO spaces, Hodge decomposition.

\section{Introduction}

We consider the linear elliptic system

$$
\operatorname{div} A(x) D u(x)=\operatorname{div} F(x)+g
$$

in a bounded domain $\Omega \subset \mathbb{R}^{n}, n>2$, with $A(x)=\left(A_{i j}(x)\right)$ symmetric, positive definite matrix with measurable coefficients, $F$ given matrix field in $W^{1,2}\left(\Omega, \mathbb{R}^{n \times n}\right)$ and $g$ given vector field in $L^{2}\left(\Omega, \mathbb{R}^{n}\right)$. A vector field $u$ in the Sobolev space $W_{0}^{1,2}\left(\Omega, \mathbb{R}^{n}\right)$ is a weak solution of the Dirichlet problem:

$$
\begin{cases}\operatorname{div} A(x) D u=\operatorname{div} F+g & \text { in } \quad \Omega \\ u=0 & \text { on } \partial \Omega\end{cases}
$$

G. Moscariello is member of Gruppo Nazionale per l'Analisi Matematica, la Probabilitá e le loro Applicazioni (GNAMPA) of INdAM. Her research has been partially supported by the National Research Project PRIN "Gradient flows, Optimal Transport and Metric Measure Structures", code 2017TEXA3H. 
if it verifies

$$
\begin{aligned}
& \int_{\Omega}\langle A(x) D u(x), D \varphi(x)\rangle d x=\int_{\Omega}\langle F(x), D \varphi(x)\rangle d x+\int_{\Omega}\langle g(x), \varphi\rangle d x \\
& \quad \forall \varphi \in C_{0}^{\infty}\left(\Omega, \mathbb{R}^{n}\right) .
\end{aligned}
$$

We assume that the entries of the matrix $A$ lie in the John - Nirenberg space $B M O$ and that for a.e. $x \in \Omega$ the following condition holds

$$
\left|A\left(x+h e_{i}\right)-A(x)\right| \leq K(x)|h|, \quad i=1, \ldots, n,
$$

with $K(x) \in L^{n, \infty}(\Omega)$, and $h \in \mathbb{R}$ such that $x+h e_{i} \in \Omega$. Thanks to a characterization of the Sobolev functions due to Hajlasz [20], the function $K$ above plays the role of the derivative $D_{x} A$. In fact this condition describes a weak form of continuity since the function $K$ may blow up at some points.

In the account of the typical functions of $B M O$ and $L^{n, \infty}$ respectively, it's obvious that the matrix

$$
A(x)=\Lambda\left(e^{-|x|}-\log |x|\right) I,
$$

with $x \in B(0,1)=\left\{x \in \mathbb{R}^{n}: 0<|x|<1\right\}$, satisfies the assumptions above. Here $\Lambda$ is a positive constant and $I$ denotes the identity matrix.

Note that the hypothesis $A(x)$ in $B M O$ guarantees that the problem (1.1) admits a unique solution (see Theorem 1.1 of [28]).

The regularity results for linear systems with continuous coefficients can be considered classical. The first remarkable contribution is due to Agmon, Douglis and Nirenberg (see [2] and [3]). Later regularity results of Schauder type in the class of Hölderian functions are proved by Campanato [8] and Morrey [26]. See also [9]. A full discussion can be found in [15] and [16].

The aim of this paper is to study the second order regularity of the solution of (1.1). More precisely, we prove the following:

Theorem 1.1. Let $\Omega$ be a regular Lipschitz domain. There exists $\varepsilon_{0}>0$, depending on $n$, such that, if

$$
\mathscr{D}_{K} \equiv \operatorname{dist}_{L^{n, \infty}}\left(K(x), L^{\infty}\right)<\varepsilon_{0},
$$

then $u \in W_{l o c}^{2,2}\left(\Omega, \mathbb{R}^{n}\right)$ and

$$
\int_{B_{R}}\left|D^{2} u\right|^{2} d x \leq c \int_{B_{2 R}}\left(\left(1+\frac{1}{R^{2}}\right)|D u|^{2}+\frac{1}{R^{2}}|F|^{2}+|D F|^{2}+|g|^{2}\right) d x,
$$

for every ball $B_{2 R} \subset \subset \Omega$ and for a constant $c$, depending on $n, \mathscr{D}_{K}$ and the $B M O$ norm of $A$.

For the definition of regular domain, see the Sect. 2 below. Anyway, balls and cubes of $\mathbb{R}^{n}$ are regular domains.

The condition (1.5) on the distance $\mathscr{D}_{K}$ of $K(x)$ to $L^{\infty}$ is clearly satisfied if the derivatives of $A(x)$ belong to any subspace of $L^{n, \infty}$ in which $L^{\infty}$ is dense, and then, in particular, if they belong to $L^{n, q}$ with $1<q<\infty$, since their distances to $L^{\infty}$ are null. On the contrary, $L^{\infty}$ is not dense in $L^{p, \infty}$ for any $p>1$. We point out that the condition (1.5) does not imply the smallness of the norm of $K(x)$ in $L^{n, \infty}$. In fact, if $A(x)$ is the matrix in (1.4), an elementary calculation shows that 
it reduces to consider the constant $\Lambda<\varepsilon_{0} \omega_{n}^{-1 / n}$, where $\omega_{n}$ denotes the measure of unit ball in $\mathbb{R}^{n}$. A value of $\varepsilon_{0}$ is given in (3.13). It follows that assuming (1.5) is more general than considering a condition on the norm and allows us to present different settings of our result in a unified way. We explicitly remark that, thanks to the embedding theorem 2.2, our result applies if the entries of $A(x)$ lie in $W^{1, n}$. The boundedness of the coefficients in a system of the type (1.1) is sometimes too restrictive in applications, as for example in phisical process of diffusion or in mathematical finance.

The novelty of Theorem 1.1 is to consider systems with $B M O$ coefficients, which feature is that they are allowed to be very irregular. In this case the energy functional

$$
\int_{\Omega}\langle A(x) D u, D u\rangle d x
$$

could not be bounded, then a priori we cannot use test functions in (1.3) proportional to the solution $u$. The Hodge decomposition and a generalization of Coifman, Rochberg and Weiss commutator result [12] allow us to establish an a priori estimate. Then the result follows by considering regularized approximating problems. If the boundary of $\Omega$ is more regular, a global version of Theorem 1.1 is also available (Proposition 4.1).

The study of the second order regularity of solutions to linear equations with discontinuous coefficients goes back to C. Miranda [25], who considered the case of coefficients in $W^{1, n}$. Then a significant improvement has been given in [5] and in $[10,11]$. Linear equations having coefficients in $B M O$ with small norm have been addressed in [19]. More recently, a condition similar to (1.5) has been considered in [16] to study the $L^{p}$ - regularity of a linear Dirichlet problem. In connection with the regularity of minimizers of functionals of the Calculus of Variations [1], the study of the regularity theory for systems had a remarkable development in last years. Recently in [13] linear systems with coefficients having in some directions locally small mean oscillation have been studied. We refer to $[23,24]$ and references therein for an almost complete recent treatment.

\section{Preliminaries}

This section is devoted to notation and preliminary results useful for our aims.

\subsection{BMO Spaces}

Definition 2.1 ([7,22]). Let $\Omega$ be a cube or the entire space $\mathbb{R}^{n}$. The $B M O(\Omega)$ space consists of all functions $b$ which are integrable on every cube $Q \subset \Omega$ with sides parallel to those of $\Omega$ and satisfy:

$$
\|b\|_{*}=\sup _{Q}\left\{\frac{1}{|Q|} \int_{Q}\left|b-b_{Q}\right| d x\right\}<\infty,
$$

where $b_{Q}=\frac{1}{|Q|} \int_{Q} b(y) d y$ and $|Q|$ denotes the Lebesgue measure of $Q$. 
It is clear that the functional $\|\cdot\|_{*}$ does not define a norm since it vanishes on constant functions. However $B M O$ becomes a Banach space provided we identify functions which differ a. e. by a constant.

Bounded functions $f$ are in $B M O$. On the other hand, $B M O$ contains unbounded functions. The standard example of $B M O$ function is

$$
f(x)=\log |x|, \quad x \in B(0,1) .
$$

We also recall the following inclusion

Theorem $2.2([7])$. For any cube $Q \subset \mathbb{R}^{n}$ the following inclusion holds with continuous embedding:

$$
W^{1, n}(Q) \hookrightarrow B M O(Q)
$$

\subsection{Hodge Decomposition}

We shall now discuss briefly the Hodge decomposition of vector fields; for a more complete treatment see [21]. For a given vector field $F=\left(f^{1}, \ldots, f^{n}\right) \in L^{p}\left(\mathbb{R}^{n}, \mathbb{R}^{n}\right)$, $1<p<\infty$, the Poisson equation $\Delta u=\operatorname{div} F$ can be solved by using the Riesz transforms in $\mathbb{R}^{n}, \mathcal{R}=\left(R_{1}, \ldots, R_{n}\right)$,

$$
\nabla u=-(\mathcal{R} \otimes \mathcal{R})(F)=: \mathscr{K}(F) .
$$

Here the tensor product operator $\mathscr{K}=-\mathcal{R} \otimes \mathcal{R}=-\left[R_{i j}\right]$ is the $n \times n$ matrix of the second order Riesz transforms $R_{i j}=R_{i} \circ R_{j}, i, j=1, \ldots, n$. Notice that the range of the operator

$$
\mathscr{H}:=I d-\mathscr{K}: L^{p}\left(\mathbb{R}^{n}, \mathbb{R}^{n}\right) \rightarrow L^{p}\left(\mathbb{R}^{n}, \mathbb{R}^{n}\right)
$$

consists of the divergence free vector fields. We then arrive at the familiar Hodge decomposition of $F$

$$
F=\nabla u+H, \quad \operatorname{div} H=0 .
$$

Hence, $L^{p}$-estimates for Riesz transform yield an uniform estimate

$$
\|\nabla u\|_{L^{p}}+\|H\|_{L^{p}} \leq c(p)\|F\|_{L^{p}} .
$$

Let $\Omega \subset \mathbb{R}^{n}$ be a domain and $G=G(x, y)$ the Green's function. For $h \in C_{0}^{\infty}(\Omega)$ the integral

$$
u(x)=\int_{\Omega} G(x, y) h(y) d y
$$

defines a solution of the Poisson equation $\Delta u=h$ with $u$ vanishing on the boundary of $\Omega$. If $h$ has a divergence form, say $h=\operatorname{div} F$ with $F=\left(f^{1}, \ldots, f^{n}\right) \in C_{0}^{\infty}\left(\Omega, \mathbb{R}^{n}\right)$, then integration by parts yields

$$
u(x)=-\int_{\Omega} \nabla_{y} G(x, y) F(y) d y .
$$

Hence the gradient of $u$ is expressed by a singular integral

$$
\nabla u(x)=-\int_{\Omega} \nabla_{x} \nabla_{y} G(x, y) F(y) d y=:\left(\mathscr{K}_{\Omega} F\right)(x) .
$$

The continuity of $\mathscr{K}_{\Omega}: L^{2}\left(\Omega, \mathbb{R}^{n}\right) \rightarrow L^{2}\left(\Omega, \mathbb{R}^{n}\right)$ is easily established by interpreting $\mathscr{K}_{\Omega} F$ as the orthogonal projection of $F$ into gradient fields. 
Let $\mathcal{D}^{p}\left(\Omega, \mathbb{R}^{n}\right)$ denote the closure of the range of the gradient operator $\nabla$ : $C_{0}^{\infty}(\Omega) \rightarrow L^{p}\left(\Omega, \mathbb{R}^{n}\right), 1<p<\infty$. If $\Omega$ is smooth, then $\mathscr{K}_{\Omega}$ extends continuously to all $L^{p}\left(\Omega, \mathbb{R}^{n}\right)$ spaces. Consequently the formula $\nabla u=\mathscr{K}_{\Omega} F$ extends to all $F \in$ $L^{p}\left(\Omega, \mathbb{R}^{n}\right)$ giving a solution with $\nabla u \in \mathcal{D}^{p}\left(\Omega, \mathbb{R}^{n}\right), 1<p<\infty$.

Definition 2.3 ([21]). A domain $\Omega \subset \mathbb{R}^{n}$ will be called regular if the operator $\mathscr{K}_{\Omega}$ acts boundedly in all $L^{p}\left(\Omega, \mathbb{R}^{n}\right)$-spaces, for $1<p<\infty$.

For $\Omega$ a regular domain we introduce, as before, the operator

$$
\mathscr{H}_{\Omega}:=I d-\mathscr{K}_{\Omega}: L^{p}\left(\Omega, \mathbb{R}^{n}\right) \rightarrow L^{p}\left(\Omega, \mathbb{R}^{n}\right) .
$$

Obviously, the range of $\mathscr{H}_{\Omega}$ consists of the divergence free vector fields on $\Omega$. We have the Hodge decomposition of $F \in L^{p}\left(\Omega, \mathbb{R}^{n}\right)$,

$$
F=\nabla u+H, \quad \operatorname{div} H=0, \quad \nabla u \in \mathcal{D}^{p}\left(\Omega, \mathbb{R}^{n}\right) .
$$

We also have the uniform estimate

$$
\|\nabla u\|_{L^{p}}+\|H\|_{L^{p}} \leq c(p, \Omega)\|F\|_{L^{p}} .
$$

We now turn to commutators. We need the following definition.

Definition 2.4 ([28]). Let $k(x): \mathbb{R}^{n} \rightarrow \mathbb{R}^{n}$. We will call $k$ a Calderon-Zygmund kernel (CZ kernel) if $k$ satisfies the following properties

1. $k(x) \in C^{\infty}\left(\mathbb{R}^{n} \backslash\{0\}\right)$,

2. $k(x)$ is homogeneous of degree $-n$,

3. $\int_{\Sigma} k(x) d \sigma_{x}=0$ where $\Sigma$ is the unit sphere of $\mathbb{R}^{n}$.

Given such a kernel, one can define a bounded operator in $L^{p}$, called CalderonZygmund singular operator, as follows

$$
K f(x)=P . V .(k \star f)(x):=P . V . \int_{\mathbb{R}^{n}} k(x-y) f(y) d y .
$$

Let $\varphi \in B M O\left(\mathbb{R}^{n}\right)$ and $k$ a CZ kernel. Following [12], we define, for $f \in L^{p}\left(\mathbb{R}^{n}\right)$ $(1<p<\infty)$, the commutator of $\varphi$ and $k$ as the principal value

$$
C[\varphi, f]=\varphi(x) P . V . k \star f(x)-P . V . k \star(\varphi f)(x) .
$$

Theorem 2.5 ([12]). Under the previous assumptions on $\varphi$ and $k, C[\varphi, f]$ is well defined for $f \in L^{p}$. Moreover $C[\varphi, f]$ is a bounded operator in $L^{p}\left(\mathbb{R}^{n}\right)$, i.e. for some constant $c=c\left(n, p,\|k\|_{L^{2}}\right)$ we have

$$
\|C[\varphi, f]\|_{L^{p}} \leq c\|\varphi\|_{*}\|f\|_{L^{p}} .
$$

We will state a generalization of Theorem 2.5 in finite - dimensional normed spaces. Let $E$ be a finite-dimensional normed space of dimension $m$,

$$
T: L^{p}\left(\mathbb{R}^{n}, E\right) \mapsto L^{p}\left(\mathbb{R}^{n}, E\right), \quad 1<p<\infty,
$$

a Calderon - Zygmund integral operator. Fixed a basis in $E$, we can associate to the operator $T$ a $m \times m$ matrix of Calderon-Zygmund operators

$$
T_{i j}: L^{p}\left(\mathbb{R}^{n}\right) \mapsto L^{p}\left(\mathbb{R}^{n}\right) .
$$


Given $A \in B M O\left(\mathbb{R}^{n}, A u t(E)\right)$, where $A u t(E)$ denotes the space of linear maps from $E$ to $E$, and

$$
\|A\|_{*}=\sup _{Q} \frac{1}{|Q|} \int_{Q}\left\|A-A_{Q}\right\| d x
$$

is the $B M O$ norm, we consider the range of $A$ :

$$
\mathcal{A}=\left\{A(z) ; z \in \mathbb{R}^{n}\right\} \subset \operatorname{Aut}(E) .
$$

Let us state the theorem:

Theorem 2.6 ([28]). Given $f \in L^{p}\left(\mathbb{R}^{n}, E\right)$ such that commutes with the range of A,

$$
T[A(z) f](x)=A(z) T f(x)
$$

for almost every $z \in \mathbb{R}^{n}$, then

$$
\|T(A f)-A T f\|_{L^{p}} \leq c\|A\|_{*}\|f\|_{L^{p}} .
$$

Since we would apply Theorem 2.6 to the projection $T$ onto divergence free matrix fields, we need to identify the range of the compatibility condition (2.2). Take now $A^{0}$ a $n \times n$-matrix, $A^{0}$ induces a linear transformation given by $A^{0}(X)=A^{0} X$, the row by column product of matrices. It is easy to verify that $A^{0}$ commutes with the operator $T$; in fact, multiplying the decomposition of $F$ by $A^{0}$ we get

$$
A^{0} F=A^{0} D u+A^{0} H=D\left(A^{0} u\right)+A^{0} H \quad \text { and } \quad \operatorname{div}\left(A^{0} H\right)=0
$$

in the sense of distributions:

$$
\int\left\langle A^{0} H, D \varphi\right\rangle=\int\left\langle H, A^{0 t} D \varphi\right\rangle=\int\left\langle H, D\left(A^{0 t} \varphi\right)\right\rangle,
$$

where $A^{0 t}$ denotes the transpose of $A^{0}$. It can be shown a local version of Theorem 2.5 in the case of Hodge decomposition of matrix fields. Therefore, given any $B M O$-matrix field $A(x)$, we get:

Lemma 2.7. ([28]) For $\Omega=\mathbb{R}^{n}$ or $\Omega$ regular domain, let $T$ be the projection onto divergence free matrix fields, and $A(x)$ a BMO - matrix field. Then

$$
\|T A(F)-A T(F)\|_{L^{p}} \leq c\|A\|_{*}\|F\|_{L^{p}}
$$

for every $F \in L^{p}\left(\Omega, R^{n \times n}\right)$.

\subsection{Lorentz Spaces}

Let $\Omega$ be a bounded domain in $\mathbb{R}^{n}$. Given $1<p, q<\infty$, the Lorentz space $L^{p, q}(\Omega)$ consists of all measurable functions $g$ defined on $\Omega$ for which the quantity

$$
\|g\|_{L^{p, q}}^{q}=p \int_{0}^{\infty}\left|\Omega_{t}(g)\right|^{\frac{q}{p}} t^{q-1} d t
$$

is finite, where $\Omega_{t}(g)=\{x \in \Omega:|g(x)|>t\}$ and $\left|\Omega_{t}\right|$ is the Lebesgue measure of $\Omega_{t}$. Note that $\|\cdot\|_{L^{p, q}}$ is equivalent to a norm and $L^{p, q}$ becomes a Banach space when endowed with it. For $p=q$, the Lorentz space $L^{p, p}$ reduces to the standard 
Lebesgue space $L^{p}$. For $q=\infty$, the class $L^{p, \infty}$ consists of all measurable functions $g$ defined on $\Omega$ such that

$$
\|g\|_{L^{p, \infty}}^{p}=\sup _{t>0} t^{p}\left|\Omega_{t}(g)\right|<\infty
$$

and it coincides with the Marcinkiewicz class, weak- $L^{p}$. For Lorentz spaces the following inclusions hold

$$
L^{r}(\Omega) \subset L^{p, q}(\Omega) \subset L^{p, r}(\Omega) \subset L^{p, \infty}(\Omega) \subset L^{q}(\Omega),
$$

whenever $1 \leq q<p<r \leq \infty$

Fundamental to us will be the Sobolev embedding theorem in Lorentz spaces (see [4]).

Theorem 2.8. Let us assume that $1<p<n, 1 \leq q \leq p$, then any function $u \in$ $W_{0}^{1,1}(\Omega)$ such that $|\nabla u| \in L^{p, q}(\Omega)$ actually belongs to $L^{p^{*}, q}(\Omega)$ and

$$
\|u\|_{L^{p^{*}, q}} \leq S_{n, p}\|\nabla u\|_{L^{p, q}}
$$

Here $p^{*}=\frac{n p}{n-p}$ and $S_{n, p}=\omega_{n}^{-\frac{1}{n}} \frac{p}{n-p}$, where $\omega_{n}$ is the Lebesgue measure of the unit ball in $\mathbb{R}^{n}$.

We define the distance of a given $f \in L^{p, \infty}$ to $L^{\infty}$ as

$$
\operatorname{dist}_{L^{p, \infty}}\left(f, L^{\infty}\right)=\inf _{g \in L^{\infty}}\|f-g\|_{L^{p, \infty}} .
$$

To find a formula for the distance, we consider the truncation operator. For $k>0$ and $y \in \mathbb{R}$, we set

$$
T_{k}(y)=\min \{k, \max \{-k, y\}\}
$$

Then

$$
\operatorname{dist}_{L^{p, \infty}}\left(f, L^{\infty}\right)=\lim _{k \rightarrow \infty}\left\|f-T_{k} f\right\|_{L^{p, \infty}} .
$$

Indeed, $\forall g \in L^{\infty}, \forall k \geq\|g\|_{L^{\infty}}$, we have for almost every $x \in \Omega$,

$$
|f(x)-g(x)| \geq\left|f(x)-T_{k} f(x)\right| .
$$

Let $\Omega$ be the unit ball of $\mathbb{R}^{n}$. The function

$$
f(x)=\frac{1}{|x|}
$$

belongs to $L^{n, \infty}$. Setting $\omega_{n}=|\Omega|$, we have

$$
\left\|f-T_{k} f\right\|_{L^{p, \infty}}=\omega_{n}^{1 / n}
$$

and it does not depend on $k$. For more details, see [15].

We recall the following relevant properties.

Lemma 2.9 ([6]). If $E \in L^{p, \infty}\left(\mathbb{R}^{n}\right), 1<p<\infty$, and $f \in L^{1}\left(\mathbb{R}^{n}\right)$, then $E \star f \in$ $L^{p, \infty}\left(\mathbb{R}^{n}\right)$ and

$$
\|E \star f\|_{L^{p, \infty}} \leq\|E\|_{L^{p, \infty}}\|f\|_{L^{1}} .
$$


Theorem 2.10. (Hölder's inequality in Lorentz spaces, [27]) If $0<p_{1}, p_{2}, p<\infty$ and $0<q_{1}, q_{2}, q \leq \infty$ obey $\frac{1}{p}=\frac{1}{p_{1}}+\frac{1}{p_{2}}$ and $\frac{1}{q}=\frac{1}{q_{1}}+\frac{1}{q_{2}}$ then

$$
\|f g\|_{L^{p, q}} \leq\|f\|_{L^{p_{1}, q_{1}}}\|g\|_{L^{p_{2}, q_{2}}}
$$

whenever the right-hand side norms are finite.

\subsection{Difference Quotients}

Definition 2.11 ([18]). Let $f(x)$ be a function defined in an open set $\Omega \subset \mathbb{R}^{n}$, and let $h$ be a real number. We call the difference quotient of $f$ with respect to $x_{s}$ the function

$$
\Delta_{s, h} f(x)=\frac{f\left(x+h e_{s}\right)-f(x)}{h} \equiv \frac{\tau_{s, h} f(x)}{h},
$$

where $e_{s}$ denotes the direction of the $x_{s}$ axis and $\tau_{s, h}$ is the finite difference operator.

When no confusion can arise, we shall omit the index $s$, and we shall write simply $\Delta_{h}$ instead of $\Delta_{s, h}$.

The function $\Delta_{s, h} f$ is defined in the set

$$
\Delta_{s, h} \Omega:=\left\{x \in \Omega: x+h e_{s} \in \Omega\right\},
$$

and hence in the set

$$
\Omega_{|h|}=\{x \in \Omega: \operatorname{dist}(x, \partial \Omega)>|h|\} .
$$

The following properties of the difference quotients are immediate:

- If $f \in W^{1, p}(\Omega)$, then $\Delta_{h} f \in W^{1, p}\left(\Omega_{|h|}\right)$, and

$$
D_{i}\left(\Delta_{h} f\right)=\Delta_{h}\left(D_{i} f\right) .
$$

- If at least one of the functions $f$ or $g$ has support contained in $\Omega_{|h|}$, then

$$
\int_{\Omega} f \Delta_{h} g d x=-\int_{\Omega} g \Delta_{-h} f d x .
$$

- We have

$$
\Delta_{h}(f g)(x)=f\left(x+h e_{s}\right) \Delta_{h} g(x)+g(x) \Delta_{h} f(x) .
$$

Remark 2.12. It follows immediately from (2.3) that the derivatives $D_{s} g$ of a Lipschitz-continuous function $g$, which exist almost everywhere as limits of the difference quotient $\Delta_{s, h} g$, coincide with its weak derivatives. In fact, if $f$ is a test function, we can pass to the limit in (2.3), getting

$$
\int f D_{s} g d x=-\int g D_{s} f d x .
$$

In other words, we have $\operatorname{Lip}(\Omega)=W^{1, \infty}(\Omega)$.

For $R>0$ and $x_{0} \in \mathbb{R}^{n}$, we define

$$
B_{R}\left(x_{0}\right)=\left\{x \in \mathbb{R}^{n}:\left|x-x_{0}\right|<R\right\},
$$

but in the case no ambiguity arises, we shall use the short notation $B_{R}$. 
Lemma 2.13. ([18]) There exists a constant $c(n)$ such that if $v \in W^{1, p}(\Omega), \Sigma \subset \subset \Omega$, $1<p<\infty, s \in\{1, \ldots, n\}$ and $|h|<h_{0}=\frac{1}{10 \sqrt{n}} \operatorname{dist}(\Sigma, \partial \Omega)$

$$
\left\|\Delta_{s, h} v\right\|_{L^{p}(\Sigma)} \leq c\left\|D_{s} v\right\|_{L^{p}(\Omega)} .
$$

Moreover, if $0<\varrho<R,|h|<R-\varrho$,

$$
\int_{B_{\varrho}}\left|v\left(x+h e_{s}\right)\right|^{p} d x \leq c(n, p) \int_{B_{R}}|v(x)|^{p} d x .
$$

Finally we recall the following useful lemma:

Lemma 2.14. ( $[17])$ For $R_{0}<R_{1}$, consider a bounded function $f:\left[R_{0}, R_{1}\right] \rightarrow$ $[0, \infty)$ with

$$
f(s) \leq \vartheta f(t)+\frac{A}{(s-t)^{\alpha}}+B \quad \text { for all } R_{0}<s<t<R_{1},
$$

where $A, B$ and $\alpha$ denote non-negative constants and $\vartheta \in(0,1)$. Then we have

$$
f\left(R_{0}\right) \leq c(\alpha, \vartheta)\left(\frac{A}{\left(R_{1}-R_{0}\right)^{\alpha}}+B\right) .
$$

\section{A Priori Estimate}

Theorem 3.1. Let $\Omega$ be a regular Lipschitz domain. If the solution $u$ of (1.2) is in $W_{\text {loc }}^{2,2}\left(\Omega, \mathbb{R}^{n}\right)$, then there exists $\varepsilon_{0}>0$, depending only on $n$, such that, if $\mathscr{D}_{K}<\varepsilon_{0}$, the following estimate holds

$$
\int_{B_{R}}\left|D^{2} u\right|^{2} d x \leq c \int_{B_{2 R}}\left(\left(1+\frac{1}{R^{2}}\right)|D u|^{2}+\frac{1}{R^{2}}|F|^{2}+|D F|^{2}+|g|^{2}\right) d x,
$$

for all $B_{2 R} \subset \subset \Omega$ and for a constant $c$ depending on $n, \mathscr{D}_{K}$ and the BMO-norm of A.

Proof. If $u \in W_{0}^{1,2}(\Omega)$ is the solution of (1.2), then for every $\varepsilon>0$ we have that $A(x) D u \in L^{2-\varepsilon}\left(\Omega, \mathbb{R}^{n \times n}\right)$ since $A$ belongs to $L^{p}$ for every $1<p<\infty$. We decompose as in (2.1)

$$
A D u=D \Psi+H,
$$

with $D \Psi \in L^{2-\varepsilon}\left(\Omega, \mathbb{R}^{n \times n}\right)$ and $H \in L^{2-\varepsilon}\left(\Omega, \mathbb{R}^{n \times n}\right)$ divergence free vector field. Since $u$ solves problem (1.2), we get

$$
\operatorname{div} D \Psi=\Delta \Psi=\operatorname{div} F+g,
$$

then, by the classical theory, $D \Psi \in L^{2}\left(\Omega, \mathbb{R}^{n \times n}\right)$ and

$$
\|D \Psi\|_{L^{2}} \leq\|F\|_{L^{2}}+\|g\|_{L^{2}} .
$$

Let us examine the other term of the Hodge decomposition: $H$ in (3.1) is a commutator with $B M O$-matrix of a gradient field in $L^{2}$; using Lemma 2.7 we conclude that $H \in L^{2}$ and

$$
\|H\|_{L^{2}} \leq c|| A\left\|_{*}\right\| D u \|_{L^{2}}
$$


where $c=c(n)$. Finally from (3.1) we deduce that $A(x) D u$ belongs to $L^{2}\left(\Omega, \mathbb{R}^{n \times n}\right)$ and we get

$$
\|A(x) D u\|_{L^{2}} \leq c(n)\left(\|A\|_{*}\|D u\|_{L^{2}}+\|F\|_{L^{2}}+\|g\|_{L^{2}}\right) .
$$

Now for a fixed ball $B_{2 R} \subset \subset \Omega$ and radii $R<s<t<2 R$ with $R$ small enough, consider a function $\xi \in C_{0}^{\infty}\left(B_{t}\right), 0 \leq \xi \leq 1, \xi=1$ on $B_{s},|\nabla \xi| \leq \frac{1}{t-s}$ and set $\psi=\xi^{2} \tau_{h} u$ for sufficiently small $h$. Since $u$ is a weak solution of (1.1), we are able to use $\varphi=\tau_{-h} \psi$ as test function in (1.3). Then

$$
\int_{B_{t}}\langle A(x) D u, D \varphi\rangle d x=\int_{B_{t}}\langle F(x), D \varphi\rangle d x+\int_{B_{t}}\langle g(x), \varphi\rangle d x,
$$

and by virtue of the properties of difference quotients

$$
\int_{B_{t}}\left\langle\tau_{h}(A(x) D u), D\left(\xi^{2} \tau_{h} u\right)\right\rangle d x=\int_{B_{t}}\left\langle\tau_{h} F(x), D \psi\right\rangle d x-\int_{B_{t}}\langle g(x), \varphi\rangle d x .
$$

It follows that

$$
\begin{gathered}
\int_{B_{t}} \xi^{2}\left\langle\tau_{h}(A(x) D u), D \tau_{h} u\right\rangle d x+2 \int_{B_{t}} \xi\left\langle\tau_{h}(A(x) D u), \nabla \xi \otimes \tau_{h} u\right\rangle d x \\
=\int_{B_{t}}\left\langle\tau_{h} F, D \psi\right\rangle d x-\int_{B_{t}}\left\langle g, \tau_{-h}\left(\xi^{2} \tau_{h} u\right)\right\rangle d x
\end{gathered}
$$

We remark that

$$
\tau_{h}(A(x) D u)=A\left(x+h e_{i}\right) D \tau_{h} u+\left(\tau_{h} A(x)\right) D u .
$$

Then from (3.2) we get

$$
\begin{aligned}
\int_{B_{t}} & \xi^{2}\left\langle A\left(x+h e_{i}\right) D \tau_{h} u, D \tau_{h} u\right\rangle d x \\
= & -\int_{B_{t}} \xi^{2}\left\langle\left(\tau_{h} A(x)\right) D u, D \tau_{h} u\right\rangle d x \\
& -2 \int_{B_{t}} \xi\left\langle A\left(x+h e_{i}\right) D \tau_{h} u, \nabla \xi \otimes \tau_{h} u\right\rangle d x \\
& -2 \int_{B_{t}} \xi\left\langle\left(\tau_{h} A(x)\right) D u, \nabla \xi \otimes \tau_{h} u\right\rangle d x \\
& +\int_{B_{t}}\left\langle\tau_{h} F, D \psi\right\rangle d x \\
& -\int_{B_{t}}\left\langle g, \tau_{-h}\left(\xi^{2} \tau_{h} u\right)\right\rangle d x \\
= & I_{1}+I_{2}+I_{3}+I_{4}+I_{5} .
\end{aligned}
$$

Now let $K_{0} \in L^{\infty}(\Omega)$. The use of Hölder's inequality in Lorentz spaces (Theorem 2.10), together with Young's inequality with a constant $\nu \in(0,1)$ that will be chosen later, yields 


$$
\begin{aligned}
\left|I_{1}\right| \leq & \int_{B_{t}} \xi^{2}|h| K(x)|D u|\left|D \tau_{h} u\right| d x \\
\leq & \frac{\nu}{2} \int_{B_{t}} \xi^{2}\left|D \tau_{h} u\right|^{2} d x+\frac{1}{2 \nu} \int_{B_{t}}|h|^{2}\left|K(x)-K_{0}\right|^{2}|\xi D u|^{2} d x \\
& +\int_{B_{t}} \xi^{2}|| K_{0}\left|L_{L^{\infty}}\right| h|| D u|| D \tau_{h} u \mid d x \\
\leq & \frac{\nu}{2} \int_{B_{t}} \xi^{2}\left|D \tau_{h} u\right|^{2} d x+\frac{|h|^{2}}{2 \nu}|| K(x)-K_{0}\left\|_{L^{n, \infty}\left(B_{t}\right)}^{2}|| \xi D u\right\|_{L^{2^{*}, 2}\left(B_{t}\right)}^{2} \\
& +\frac{\nu}{2} \int_{B_{t}} \xi^{2}\left|D \tau_{h} u\right|^{2} d x+\frac{|h|^{2}}{2 \nu} \int_{B_{t}} \xi^{2}\left\|K_{0}\right\|_{L^{\infty}}^{2}|D u|^{2} d x .
\end{aligned}
$$

Finally by Theorem 2.8

$$
\begin{aligned}
\left|I_{1}\right| \leq \nu & \int_{B_{t}} \xi^{2}\left|D \tau_{h} u\right|^{2} d x+ \\
& +\frac{|h|^{2}}{2 \nu} S_{2, n}^{2}\left\|K(x)-K_{0}\right\|_{L^{n, \infty}}^{2}\|D(\xi D u)\|_{L^{2}\left(B_{t}\right)}^{2}+ \\
& +\frac{|h|^{2}}{2 \nu} \int_{B_{t}} \xi^{2}\left\|K_{0}\right\|_{L^{\infty}}^{2}|D u|^{2} d x .
\end{aligned}
$$

Next we estimate $I_{2}$.

$$
\begin{aligned}
\left|I_{2}\right| & \leq 2 \int_{B_{t}-B_{s}}|\xi|\left|A\left(x+h e_{i}\right) \tau_{h} u\right|\left|D \tau_{h} u\right||\nabla \xi| d x \\
& \leq \int_{B_{t}-B_{s}} \xi^{2}\left|D \tau_{h} u\right|^{2} d x+\int_{B_{t}-B_{s}}|\nabla \xi|^{2}\left|A\left(x+h e_{i}\right) \tau_{h} u\right|^{2} d x \\
& \leq \int_{B_{t}-B_{s}} \xi^{2}\left|D \tau_{h}(u)\right|^{2} d x+\frac{1}{(t-s)^{2}} \int_{B_{t}-B_{s}}\left|A\left(x+h e_{i}\right) \tau_{h} u\right|^{2} d x
\end{aligned}
$$

Now we estimate $I_{3}$. Again by Hölder's and Young's inequalities, we get

$$
\begin{aligned}
\left|I_{3}\right| \leq & 2 \int_{B_{t}}|\xi|\left|A\left(x+h e_{i}\right)-A(x)\right||D u||\nabla \xi|\left|\tau_{h} u\right| d x \\
\leq & \int_{B_{t}}|\xi|^{2}\left|A\left(x+h e_{i}\right)-A(x)\right|^{2}|D u|^{2} d x+\int_{B_{t}-B_{s}}|\nabla \xi|^{2}\left|\tau_{h} u\right|^{2} d x \\
\leq & 2 \int_{B_{t}}|h|^{2}|\xi|^{2}\left|K(x)-K_{0}\right|^{2}|D u|^{2} d x+2 \int_{B_{t}}|h|^{2}|\xi|^{2}|| K_{0} \|_{L^{\infty}}^{2}|D u|^{2} d x \\
& +\int_{B_{t}-B_{s}}|\nabla \xi|^{2}\left|\tau_{h} u\right|^{2} d x \\
\leq & 2|h|^{2}|| K(x)-\left.K_{0}\right|_{L^{n, \infty}} ^{2}|| \xi D u\left\|_{L^{2^{*}, 2}}^{2}+2|h|^{2}|| K_{0}\right\|_{L^{\infty}}^{2} \int_{B_{t}} \xi^{2}|D u|^{2} d x \\
& +\frac{1}{(t-s)^{2}} \int_{B_{t}-B_{s}}\left|\tau_{h} u\right|^{2} d x .
\end{aligned}
$$


Applying Theorem 2.8, then

$$
\begin{aligned}
\left|I_{3}\right| \leq & 4 S_{2, n}^{2}|h|^{2}\left\|K(x)-K_{0}\right\|_{L^{n, \infty}}^{2}\left\|\xi D^{2} u\right\|_{L^{2}}^{2}+ \\
& +2|h|^{2}\left\|K_{0}\right\|_{L^{\infty}}^{2} \int_{B_{t}} \xi^{2}|D u|^{2} d x+ \\
& +\frac{1}{(t-s)^{2}} \int_{B_{t}-B_{s}}\left|\tau_{h} u\right|^{2} d x+ \\
& +4|h|^{2} S_{2, n}^{2} \frac{1}{(t-s)^{2}}|| K(x)-K_{0} \|_{L^{n, \infty}}^{2} \int_{B_{t}-B_{s}}|D u|^{2} d x
\end{aligned}
$$

Finally we estimate $I_{4}$ :

$$
\begin{aligned}
\left|I_{4}\right|= & \left|\int_{B_{t}}\left\langle\tau_{h} F, D\left(\xi^{2} \tau_{h} u\right)\right\rangle d x\right| \\
\leq & \frac{\nu}{2} \int_{B_{t}} \xi^{2}\left|D \tau_{h} u\right|^{2} d x+\frac{1}{2 \nu} \int_{B_{t}} \xi^{2}\left|\tau_{h} F\right|^{2} d x \\
& +\int_{B_{t}} \xi^{2}\left|\tau_{h} F\right|^{2} d x+\int_{B_{t}}|\nabla \xi|^{2}\left|\tau_{h} u\right|^{2} d x \\
\leq & \frac{\nu}{2} \int_{B_{t}} \xi^{2}\left|D \tau_{h} u\right|^{2} d x+\left(\frac{1}{2 \nu}+1\right) \int_{B_{t}} \xi^{2}\left|\tau_{h} F\right|^{2} d x \\
& +\frac{1}{(t-s)^{2}} \int_{B_{t}-B_{s}}\left|\tau_{h} u\right|^{2} d x,
\end{aligned}
$$

and $I_{5}$ :

$$
\begin{aligned}
\left|I_{5}\right|= & \left|h \int_{B_{t}} g \frac{\tau_{-h}\left(\xi^{2} \tau_{h} u\right)}{h} d x\right| \\
\leq & \frac{1}{2 \nu}|h|^{2} \int_{B_{t}}|g(x)|^{2} d x+\frac{\nu}{2} \int_{B_{t}}\left|\frac{\tau_{-h}\left(\xi^{2} \tau_{h} u\right)}{h}\right|^{2} d x \\
\leq & \frac{1}{2 \nu}|h|^{2} \int_{B_{t}}|g(x)|^{2} d x+\frac{\nu}{2} \int_{B_{t}}\left|\frac{\tau_{-h}\left(\xi^{2}\right)\left(-\tau_{-h} u\right)+\xi^{2} \tau_{-h}\left(\tau_{h} u\right)}{h}\right|^{2} d x \\
\leq & \frac{1}{2 \nu}|h|^{2} \int_{B_{t}}|g(x)|^{2} d x+\nu \int_{B_{t}}\left|\tau_{-h}\left(\xi^{2}\right) \frac{\tau_{-h} u}{h}\right|^{2} d x \\
& +\nu \int_{B_{t}} \xi^{4}\left|\frac{\tau_{-h}\left(\tau_{h} u\right)}{h}\right|^{2} d x \\
= & \frac{1}{2 \nu}|h|^{2} \int_{B_{t}}|g(x)|^{2} d x+\nu|h|^{2} \int_{B_{t}}\left|\frac{\tau_{-h}\left(\xi^{2}\right)}{h}\right|^{2}\left|\frac{\tau_{-h} u}{h}\right|^{2} d x \\
& +\nu \int_{B_{t}} \xi^{4}\left|\frac{\tau_{-h}\left(\tau_{h} u\right)}{h}\right|^{2} d x .
\end{aligned}
$$


Combining estimates (3.3)-(3.8), we get

$$
\begin{aligned}
& \int_{B_{t}} \xi^{2}\left|D \tau_{h} u\right|^{2} d x \leq \frac{3}{2} \nu \int_{B_{t}} \xi^{2}\left|D \tau_{h} u\right|^{2} d x \\
& +\left(\frac{1}{\nu}+4\right)|h|^{2} S_{2, n}^{2}|| K(x)-\left.K_{0}\right|_{L^{n, \infty}} ^{2} \int_{B_{t}}\left|\xi D^{2} u\right|^{2} d x \\
& \quad+\frac{1}{(t-s)^{2}} \int_{B_{t}}\left|A\left(x+h e_{i}\right) \tau_{h} u\right|^{2} d x+\left(2+\frac{1}{2 \nu}\right)|h|^{2}|| K_{0} \|_{L^{\infty}}^{2} \int_{B_{t}} \xi^{2}|D u|^{2} d x \\
& +\frac{2}{(t-s)^{2}} \int_{B_{t}-B_{s}}\left|\tau_{h} u\right|^{2} d x+\left(1+\frac{1}{2 \nu}\right) \int_{B_{t}} \xi^{2}\left|\tau_{h} F\right|^{2} d x+\int_{B_{t}-B_{s}} \xi^{2}\left|D \tau_{h} u\right|^{2} d x \\
& +|h|^{2}\left(\frac{1}{\nu}+4\right)|| K-K_{0}||_{L^{n, \infty}} S_{2, n}^{2} \frac{1}{(t-s)^{2}} \int_{B_{t}-B_{s}}|D u|^{2} d x \\
& \quad+\frac{1}{2 \nu}|h|^{2} \int_{B_{t}}|g(x)|^{2} d x+\left.\nu|h|^{2} \int_{B_{t}}\left|\frac{\tau_{-h}\left(\xi^{2}\right)}{h}\right| \frac{\tau_{-h} u}{h}\right|^{2} d x \\
& +\nu \int_{B_{t}} \xi^{4}\left|\frac{\tau_{-h}\left(\tau_{h} u\right)}{h}\right|^{2} d x .
\end{aligned}
$$

Next we divide by $|h|^{2}$ in (3.9) and, by Lemma 2.13, as $h \rightarrow 0^{+}$, we get

$$
\begin{aligned}
& \left(1-\frac{5}{2} \nu\right) \int_{B_{t}} \xi^{2}\left|D^{2} u\right|^{2} d x \leq\left(\frac{1}{\nu}+4\right) S_{2, n}^{2}|| K-K_{0} \|_{L^{n, \infty}}^{2} \int_{B_{t}} \xi^{2}\left|D^{2} u\right|^{2} d x \\
& \quad+\int_{B_{t}-B_{s}} \xi^{2}\left|D^{2} u\right|^{2} d x+\frac{1}{(t-s)^{2}} \int_{B_{t}}|A(x) D u|^{2} d x \\
& \quad+\left(\frac{1}{2 \nu}+2\right)|| K_{0} \|_{L^{\infty}}^{2} \int_{B_{t}} \xi^{2}|D u|^{2} d x+\frac{2}{(t-s)^{2}} \int_{B_{t}-B_{s}}|D u|^{2} d x \\
& \quad+\frac{4 \nu}{(t-s)^{2}} \int_{B_{t}}|D u|^{2} d x+\left(\frac{1}{2 \nu}+1\right) \int_{B_{t}} \xi^{2}|D F|^{2} d x \\
& \quad+\left(\frac{1}{\nu}+4\right) S_{2, n}^{2}|| K-K_{0} \|_{L^{n, \infty}}^{2} \frac{1}{(t-s)^{2}} \int_{B_{t}-B_{s}}|D u|^{2} d x+\frac{1}{2 \nu} \int_{B_{t}}|g(x)|^{2} d x .
\end{aligned}
$$

Now we put $\nu=\nu_{0}:=\frac{\sqrt{65}-5}{20}$, in order to maximize the function $\frac{1-\frac{5}{2} \nu}{4+\frac{1}{\nu}}$, and $\eta:=$ $\frac{1}{S_{2, n}} \sqrt{\frac{1-\frac{5}{2} \nu_{0}}{4+\frac{1}{\nu_{0}}}}=\frac{\sqrt{9-\sqrt{65}}}{4 S_{2, n}}$. Let $\varepsilon_{0}$ be a number such that $0<\varepsilon_{0}<\eta$. If

$$
\mathscr{D}_{K}<\varepsilon_{0},
$$

then we can choose $K_{0} \in L^{\infty}(\Omega)$ such that $\left(1-\frac{5}{2} \nu_{0}-\left(\frac{1}{\nu_{0}}+4\right) S_{2, n}^{2}\left\|K-K_{0}\right\|_{L^{n, \infty}}^{2}\right)$ $>\left(1-\frac{5}{2} \nu_{0}-\left(\frac{1}{\nu_{0}}+4\right) S_{2, n}^{2} \varepsilon_{0}^{2}\right)>0$. Then, by reabsorbing the first term of the right hand side of (3.10) in the left hand side, since $\xi=1$ on $B_{s}$ and $0 \leq \xi \leq 1$, we get 


$$
\begin{aligned}
& C \int_{B_{s}}\left|D^{2} u\right|^{2} d x \leq \int_{B_{t}-B_{s}}\left|D^{2} u\right|^{2} d x+\frac{1}{(t-s)^{2}} \int_{B_{t}}|A(x) D u|^{2} d x \\
& \quad+\left(\frac{1}{2 \nu_{0}}+2\right)\left\|K_{0}\right\|_{L^{\infty}}^{2} \int_{B_{t}}|D u|^{2} d x+\left(3+\frac{3}{2} \nu_{0}\right) \frac{1}{(t-s)^{2}} \int_{B_{t}}|D u|^{2} d x \\
& \quad+\left(\frac{1}{2 \nu_{0}}+1\right) \int_{B_{t}}|D F|^{2} d x+\frac{1}{2 \nu_{0}} \int_{B_{t}}|g(x)|^{2} d x
\end{aligned}
$$

where $C=1-\frac{5}{2} \nu_{0}-\left(\frac{1}{\nu_{0}}+4\right) S_{2, n}^{2} \varepsilon_{0}^{2}$.

Now we fill the hole, having

$$
\begin{aligned}
& \int_{B_{s}}\left|D^{2} u\right|^{2} d x \leq \frac{1}{C+1} \int_{B_{t}}\left|D^{2} u\right|^{2} d x+\frac{1}{C+1}\left(\frac{1}{(t-s)^{2}} \int_{B_{t}}|A(x) D u|^{2} d x\right. \\
& \quad+\left(\frac{1}{2 \nu_{0}}+2\right)|| K_{0} \|_{L^{\infty}}^{2} \int_{B_{t}}|D u|^{2} d x+\left(3+\frac{3}{2} \nu_{0}\right) \frac{1}{(t-s)^{2}} \int_{B_{t}}|D u|^{2} d x \\
& \left.\quad+\left(\frac{1}{2 \nu_{0}}+1\right) \int_{B_{t}}|D F|^{2} d x+\frac{1}{2 \nu_{0}} \int_{B_{t}}|g(x)|^{2} d x\right) .
\end{aligned}
$$

Then by Lemma 2.14

$$
\begin{aligned}
\int_{B_{R}}\left|D^{2} u\right|^{2} d x \leq & c\left(\frac{1}{R^{2}} \int_{B_{2 R}}\left(|| A||_{*}^{2}|D u|^{2}+|F|^{2}\right) d x\right. \\
& +\left(1+\frac{1}{R^{2}}\right) \int_{B_{2 R}}|D u|^{2} d x \\
& \left.+\int_{B_{2 R}}|D F|^{2} d x+\int_{B_{2 R}}|g(x)|^{2} d x\right),
\end{aligned}
$$

where $c=c\left(n, \mathscr{D}_{K}\right)$, and therefore we have the result.

Remark 3.2. The bound in (3.11) could be not optimal. Anyway it is comparable with analogous bounds in [14].

Remark 3.3. We explicitly observe that the dependence of the constant in (3.13) on $\mathscr{D}_{K}$ occurs only through the norm of $K_{0}$ in $L^{\infty}$.

\section{Regularity}

Given a symmetric matrix-valued function $A(x) \in B M O\left(\Omega, \mathbb{R}^{n \times n}\right), \Omega$ Lipschitz domain, we assume that

$$
\langle A(x) Y, Y\rangle \geq\|Y\|^{2}
$$

for every matrix $Y \in \mathbb{R}^{n \times n}$. We consider the following system with Dirichlet boundary conditions :

$$
\begin{cases}\operatorname{div}(A D u)=\operatorname{div} F+g & \text { in } \Omega \\ u=0 & \text { on } \partial \Omega\end{cases}
$$


where $u: \Omega \mapsto \mathbb{R}^{n}$ is a $W_{0}^{1,2}$ vector-valued function, $F$ is a field in $W^{1,2}\left(\Omega, \mathbb{R}^{n \times n}\right)$ and $g$ is a field in $L^{2}\left(\Omega, \mathbb{R}^{n}\right)$. Notice that we do not require that $A$ belongs to $L^{\infty}$, the system have to be understood in the weak sense

$$
\begin{gathered}
\int_{\Omega}\langle A(x) D u(x), D \varphi(x)\rangle d x=\int_{\Omega}\langle F(x), D \varphi(x)\rangle d x+\int_{\Omega}\langle g(x), \varphi(x)\rangle d x \\
\forall \varphi \in C_{0}^{\infty}\left(\Omega, \mathbb{R}^{n}\right) .
\end{gathered}
$$

Because $A \in B M O$, we know that $A \in L^{2}$ but $A$ doesn't need to be bounded; however we know, by Theorem 1.1 in [28], that the Dirichlet problem (4.1) admits a unique solution $u$ in $W_{0}^{1,2}$.

Now we are in position to prove Theorem 1.1 .

Proof of Theorem 1.1. We first extend the matrix $A$ to $\mathbb{R}^{n}$, putting zero outside of $\Omega$. Then we take $\varrho \in C_{0}^{\infty}\left(\mathbb{R}^{n}\right)$ such that supp $\varrho \subset \overline{B_{1}(0)}, \varrho \geq 0, \varrho \neq 0$ and $\infty$, and we consider the convolution $A_{N}=A \star \varrho_{N}$, with $\varrho_{N}=\frac{N^{n} \varrho(N x)}{\int \varrho}$ and defined by:

$$
A_{N}(x)=\int_{\mathbb{R}^{n}} A(y) \varrho_{N}(x-y) d y, \quad x \in \bar{\Omega} .
$$

We notice that

1. $A_{N} \in C^{\infty}\left(\bar{\Omega}, \mathbb{R}^{n \times n}\right) \cap L^{\infty}\left(\Omega, \mathbb{R}^{n \times n}\right)$,

2. $\left\langle A_{N} Y, Y\right\rangle \geq\|Y\|^{2}$,

3. $\left|A_{N}\left(x+h e_{i}\right)-A_{N}(x)\right| \leq K_{N}(x)|h|$,

4. $K_{N}(x)=\left(K \star \varrho_{N}\right)(x) \in L^{n, \infty}$,

5. $\left\|A_{N}\right\|_{*} \leq\|A\|_{*}$,

6. $A_{N}$ converges to $A$ in $L^{2}$.

We find solutions $u_{N} \in W_{0}^{1,2}\left(\Omega, \mathbb{R}^{n}\right)$ of the Dirichlet problems :

$$
\begin{cases}\operatorname{div}\left(A_{N} D u_{N}\right)=\operatorname{div} F+g & \text { in } \Omega \\ u_{N}=0 & \text { on } \partial \Omega,\end{cases}
$$

that converge weakly in $W_{0}^{1,2}$, and strongly in $L^{2}$, to $u$ (see [28]) and it is well known that $u_{N} \in W_{l o c}^{2,2}\left(\Omega, \mathbb{R}^{n}\right)$ (see $\left.[18]\right)$.

Let $\varepsilon_{0}>0$ be the number fixed in (3.11) and let us assume

$$
\mathscr{D}_{K}<\varepsilon_{0} .
$$

We notice that, from Lemma 2.9, we have

$$
\mathscr{D}_{K_{N}} \leq \mathscr{D}_{K}<\varepsilon_{0} \text {. }
$$

More precisely, if $K_{0} \in L^{\infty}(\Omega)$ is a function such that $\left\|K-K_{0}\right\|_{L^{n, \infty}}<\varepsilon_{0}$, we get

$$
\left\|K_{N}-K_{0}\right\|_{L^{n, \infty}} \leq\left\|K-K_{0}\right\|_{L^{n, \infty}}+\left\|\left(K_{0}\right)_{N}-K_{0}\right\|_{L^{n, \infty}} .
$$

Since $K_{0} \in L^{p}(\Omega)$ for every $p \geq n$, thanks to Theorem 2.10 the second term in the right hand side of the previous inequality goes to 0 as $N \rightarrow+\infty$. Then we can assume that

$$
\left\|K_{N}-K_{0}\right\|_{L^{n, \infty}}<\varepsilon_{0}
$$

for $N$ sufficiently large. 
Now, arguing as in Theorem 3.1, we get the following relation

$$
\begin{aligned}
& \int_{B_{s}}\left|D^{2} u_{N}\right|^{2} d x \leq \frac{1}{C+1} \int_{B_{t}}\left|D^{2} u_{N}\right|^{2} d x \\
& \quad+\frac{1}{C+1}\left(\frac{1}{(t-s)^{2}} \int_{B_{t}}\left|A_{N}(x) D u_{N}\right|^{2} d x\right. \\
& \quad+\left.\left(\frac{1}{2 \nu_{0}}+2\right)|| K_{0}\right|_{L^{\infty}} ^{2} \int_{B_{t}}\left|D u_{N}\right|^{2} d x \\
& \quad+\left(3+\frac{3}{2} \nu_{0}\right) \frac{1}{(t-s)^{2}} \int_{B_{t}}\left|D u_{N}\right|^{2} d x+\left(\frac{1}{2 \nu_{0}}+1\right) \int_{B_{t}}|D F|^{2} d x \\
& \left.\quad+\frac{1}{2 \nu_{0}} \int_{B_{t}}|g(x)|^{2} d x\right)
\end{aligned}
$$

where the constant $C$ is the same of (3.12).

Applying Lemma 2.14 we deduce

$$
\begin{aligned}
\int_{B_{R}}\left|D^{2} u_{N}\right|^{2} d x \leq & c\left(n, \mathscr{D}_{K}\right)\left(\frac{1}{R^{2}} \int_{B_{2 R}}\left(|| A||_{*}^{2}\left|D u_{N}\right|^{2}+|F|^{2}\right) d x\right. \\
& +\left(1+\frac{1}{R^{2}}\right) \int_{B_{2 R}}\left|D u_{N}\right|^{2} d x \\
& \left.+\int_{B_{2 R}}|D F|^{2} d x+\int_{B_{2 R}}|g(x)|^{2} d x\right)
\end{aligned}
$$

and

$$
\int_{B_{R}}\left|D^{2} u_{N}\right|^{2} d x \leq c \int_{B_{2 R}}\left(\left(1+\frac{1}{R^{2}}\right)\left|D u_{N}\right|^{2}+\frac{1}{R^{2}}|F|^{2}+|D F|^{2}+|g|^{2}\right) d x,
$$

with $c=c\left(n, \mathscr{D}_{K},\|A\|_{*}\right)$. From the previous relation, we deduce that $\left|D u_{N}\right|$ is a bounded sequence in $W^{1,2}\left(B_{R}\right)$. Then, by compactness, up to a sequence not relabeled, we deduce that $\left|D u_{N}\right|$ converges to $|D u|$ in $L^{2}\left(B_{R}\right)$. Finally, by the semicontinuity of the norm, we get

$$
\begin{aligned}
& \int_{B_{R}}\left|D^{2} u\right|^{2} d x \leq \liminf _{N} \int_{B_{R}}\left|D^{2} u_{N}\right|^{2} d x \\
& \quad \leq c\left(n, \mathscr{D}_{K},\|A\|_{*}\right) \int_{B_{2 R}}\left(\left(1+\frac{1}{R^{2}}\right)|D u|^{2}+\frac{1}{R^{2}}|F|^{2}+|D F|^{2}+|g|^{2}\right) d x .
\end{aligned}
$$

Now we prove a global version of Theorem 1.1 .

Proposition 4.1. Let $\Omega$ be a regular domain with $C^{2}$ boundary. There exists $\varepsilon_{1}>0$, depending on $n$ and $\Omega$, such that, if

$$
\mathscr{D}_{K} \equiv \operatorname{dist}_{L^{n, \infty}}\left(K(x), L^{\infty}\right)<\varepsilon_{1},
$$

then $u \in W^{2,2}\left(\Omega, \mathbb{R}^{n}\right)$ and

$$
\int_{\Omega}\left|D^{2} u\right|^{2} d x \leq c \int_{\Omega}\left(|D u|^{2}+|F|^{2}+|D F|^{2}+|g|^{2}\right) d x
$$

for a constant $c$, depending on $n, \mathscr{D}_{K}, \Omega$ and the BMO-norm of $A$. 
Proof. We cover $\Omega$ by a family of open sets $\Omega^{\prime}, \Omega^{\prime \prime}, U_{1}, \ldots, U_{m}, V_{1}, \ldots, V_{m}$ such that

1. $\Omega^{\prime} \subset \subset \Omega^{\prime \prime} \subset \subset \Omega$;

2. $U_{l}, V_{l}$ are neighbourhoods centered in $x_{l} \in \partial \Omega$, with $l=1, \ldots, m$;

3. $V_{l} \subset \subset U_{l}$, with $l=1, \ldots, m$;

4. $\cup_{l=1}^{m} V_{l} \supsetneq \partial \Omega$;

5. $\Omega \subsetneq \cup_{l=1}^{m} V_{l} \cup \Omega^{\prime}$.

We consider the solutions $u_{N}$ of the problems (4.2). Covering $\bar{\Omega}^{\prime}$ by a finite number of balls, we have that

$$
\int_{\Omega^{\prime}}\left|D^{2} u_{N}\right|^{2} d x \leq c\left(n, \mathscr{D}_{K}, \Omega,\|A\|_{*}\right) \int_{\Omega}\left(\left|D u_{N}\right|^{2}+|F|^{2}+|D F|^{2}+|g|^{2}\right) d x .
$$

Now we focus on the boundary regularity of the solutions. Fixed $l \in\{1, \ldots, m\}$, on every $U_{l}$ we can consider the diffeomorphism $\Phi(x)=\left(\Phi_{1}(x), \ldots, \Phi_{n}(x)\right)$ which maps $\Omega_{l} \equiv U_{l} \cap \Omega$ to an open set of $\mathbb{R}^{n}$ and defined by

$$
\begin{aligned}
& \Phi_{i}(x)=x_{i}, \quad i=1, \ldots, n-1 ; \\
& \Phi_{n}(x)=x_{n}-\psi_{l}\left(x^{\prime}\right), \quad \text { with } x^{\prime}=\left(x_{1}, \ldots, x_{n-1}\right),
\end{aligned}
$$

where $\psi_{l}: \mathbb{R}^{n-1} \rightarrow \mathbb{R}$ is $C^{2}$ and whose graph coincides with $\partial \Omega$ in $U_{l} . \Phi(x)=: y$ is such that

$$
\Phi\left(U_{l} \cap \Omega\right) \subset\left\{y \in \mathbb{R}^{n}: y_{n}>0\right\}, \quad \Phi\left(U_{l} \cap \partial \Omega\right) \subset\left\{y \in \mathbb{R}^{n}: y_{n}=0\right\} .
$$

It can be seen that $\Phi$ is invertible, both $\Phi$ and $\Phi^{-1}$ are $C^{2}$ functions. Let $\tilde{u}_{N}$ be such that $u_{N}(x)=\left(\tilde{u}_{N} \circ \Phi\right)(x), x \in U_{l} \cap \bar{\Omega}$, and we check that $\tilde{u}_{N}$ solves in $\tilde{\Omega}_{l}=\Phi\left(U_{l} \cap \Omega\right)$ the system

$$
\begin{cases}\operatorname{div} \tilde{A}_{N}(x) D \tilde{u}_{N}=\operatorname{div} \tilde{F}+\tilde{g} & \text { in } \quad \tilde{\Omega}_{l} \\ \tilde{u}_{N}=0 & \text { on } \quad\left\{y_{n}=0\right\} \cap \partial \tilde{\Omega}_{l}\end{cases}
$$

where

$$
\tilde{A}_{N}=\left[D \Phi \cdot A_{N} \cdot(D \Phi)^{t}\right] \circ \Phi^{-1}, \quad \tilde{F}=(F \cdot D \Phi) \circ \Phi^{-1}, \quad \tilde{g}=g \circ \Phi^{-1} .
$$

These formulas can be derived starting from the weak formulation of the problem and applying a change of variables in order to express the different integrals in terms of the new coordinates.

For instance, for $\varphi \in C_{0}^{\infty}\left(\Omega_{l}\right)$,

$$
\int_{\Omega_{l}}\langle g(x), \varphi(x)\rangle d x=\int_{\tilde{\Omega}_{l}}\left\langle g \circ \Phi^{-1}(y), \varphi \circ \Phi^{-1}(y)\right\rangle \operatorname{det}\left(D \Phi^{-1}(y)\right) d y,
$$

just letting $x=\Phi^{-1}(y)$, but then $\operatorname{det}\left(D \Phi^{-1}\right)=1$ and we can set $\varphi=\eta \circ \Phi$ so that equivalently $\eta=\varphi \circ \Phi^{-1}$ and

$$
\int_{\Omega_{l}}\langle g(x), \varphi(x)\rangle d x=\int_{\tilde{\Omega}_{l}}\langle\tilde{g}(y), \eta(y)\rangle d y .
$$


We have to prove that the conditions on $A$ still hold true for $\tilde{A}$. About the uniform ellipticity, we have that

$$
\begin{aligned}
\left\langle\tilde{A}_{N}(y) Y, Y\right\rangle & =\left\langle A_{N}\left(\Phi^{-1}(y)\right) D \Phi\left(\Phi^{-1}(y)\right) Y, D \Phi\left(\Phi^{-1}(y)\right) Y\right\rangle \\
& \geq\left\langle D \Phi\left(\Phi^{-1}(y)\right) Y, D \Phi\left(\Phi^{-1}(y)\right) Y\right\rangle \\
& \geq c\|Y\|^{2}, \quad c>0 .
\end{aligned}
$$

Hence, $\tilde{A}_{N}$ satisfies the uniform ellipticity condition; moreover $B M O$ and $L^{n, \infty}$ are preserved under $C^{2}$ transformations. Finally, we have

$$
\left|\tilde{A}_{N}\left(y+h e_{i}\right)-\tilde{A}_{N}(y)\right| \leq \tilde{K}_{N}(y)|h|, \quad y \in \tilde{\Omega}_{l},
$$

with $\mathscr{D}_{\tilde{K}_{N}}=\mathscr{D}_{K_{N}}$.

Therefore, if $D^{\prime} \tilde{u}_{N}$ indicates any derivatives $D_{s} \tilde{u}_{N}$ with $s \neq n$, we have that $D^{\prime} \tilde{u}_{N} \in W_{l o c}^{1,2}\left(\tilde{\Omega}_{l}, \mathbb{R}^{n}\right)$ and

$$
\int_{B_{R}^{+}}\left|D D^{\prime} \tilde{u}_{N}\right|^{2} d y \leq c \int_{B_{2 R}^{+}}\left(\left(1+\frac{1}{R^{2}}\right)\left|D \tilde{u}_{N}\right|^{2}+\frac{1}{R^{2}}|\tilde{F}|^{2}+|D \tilde{F}|^{2}+|\tilde{g}|^{2}\right) d y,
$$

where $B_{R}^{+}=\left\{y=\left(y_{1}, \ldots, y_{n}\right) \in \mathbb{R}^{n}:\|y\|<R, y_{n}>0\right\}$ and $B_{2 R}^{+} \subset \subset \tilde{\Omega}_{l}$. In order to have the estimate for the derivatives $D_{n n}^{2} \tilde{u}_{N}$, the equation readily implies that $D_{n}\left(\tilde{A}_{N}^{i n} D_{n} \tilde{u}_{N}^{j}\right) \in L^{2}\left(\tilde{\Omega}_{l}\right)$ for $i, j \in\{1, \ldots, n\}$; by Lemma 2.13 the difference quotients $\Delta_{h}\left(\tilde{A}^{i n} D_{n} \tilde{u}_{N}^{j}\right)$ have uniformly bounded $L^{2}$ norm in $\tilde{\Omega}_{l_{|h|}}$ and the same is true for $\tilde{A}^{i n} \Delta_{h} D_{n} \tilde{u}_{N}^{j}$. The uniform ellipticity condition gives that $D_{n n}^{2} \tilde{u}_{N} \in L^{2}\left(\tilde{\Omega}_{l}\right)$ and the analogous estimate for $D_{n n}^{2} \tilde{u}_{N}$.

Taking the covering introduced above with $U_{l}=\Phi^{-1}\left(B_{2 R}^{+}\right)$and $V_{l}=\Phi^{-1}\left(B_{R}^{+}\right)$ and coming back to the original variables, we get that

$$
\int_{V_{l}}\left|D^{2} u_{N}\right|^{2} d x \leq c \int_{V_{l}}\left(\left|D u_{N}\right|^{2}+|F|^{2}+|D F|^{2}+|g|^{2}\right) d x
$$

and summing

$$
\int_{\Omega}\left|D^{2} u_{N}\right|^{2} d x \leq c \int_{\Omega}\left(\left|D u_{N}\right|^{2}+|F|^{2}+|D F|^{2}+|g|^{2}\right) d x .
$$

Arguing as in the proof of the Theorem 1.1, we get the desired result.

\section{Acknowledgements}

The Authors thank the referee for all valuable comments helping to concretely improve exposition of the results.

Open Access. This article is licensed under a Creative Commons Attribution 4.0 International License, which permits use, sharing, adaptation, distribution and reproduction in any medium or format, as long as you give appropriate credit to the original author(s) and the source, provide a link to the Creative Commons licence, and indicate if changes were made. The images or other third party material in this article are included in the article's Creative Commons licence, unless indicated otherwise in a credit line to the material. If material is not included in the article's Creative Commons licence and your intended use is not permitted by statutory regulation or exceeds the 
permitted use, you will need to obtain permission directly from the copyright holder. To view a copy of this licence, visit http://creativecommons.org/licenses/by/4.0/.

Publisher's Note Springer Nature remains neutral with regard to jurisdictional claims in published maps and institutional affiliations.

\section{References}

[1] Acerbi, E., Fusco, N.: Regularity of minimizers of non-quadratic functionals: the case $1<p<2$. J. Math. Anal. Appl. 140, 115-135 (1989)

[2] Agmon, S., Douglis, A., Nirenberg, L.: Estimates near the boundary for solutions of elliptic partial differential equations satisfying general boundary conditions I. Commun. Pure Appl. Math. XII, 623-727 (1959)

[3] Agmon, S., Douglis, A., Nirenberg, L.: Estimates near the boundary for solutions of elliptic partial differential equations satisfying general boundary conditions II. Commun. Pure Appl. Math. XVII, 35-92 (1964)

[4] Alvino, A.: Sulla disuguaglianza di Sobolev in Spazi di Lorentz. Boll. Un. Mat. It. A (5) 14, 148-156 (1977)

[5] Alvino, A., Trombetti, G.: Second order elliptic equations whose coefficients have their first derivatives weakly - $L^{n}$. Ann. Mat. Pura Appl. (4) 138, 331-340 (1985)

[6] Benilan, P., Brezis, H., Crandall, M.: A semilinear equation in $L^{1}\left(\mathbb{R}^{N}\right)$. Annali della Scuola Normale Superiore di Pisa, Classe di Scienze, serie 4, tomo 2 4, 523-555 (1975)

[7] Brezis, H., Nirenberg, L.: Degree theory and $B M O$; part $I$ : compact manifolds without boundaries. Selecta Mathematica New Series 1, 197-263 (1995)

[8] Campanato, S.: Equazioni ellittiche del secondo ordine e spazi $\mathcal{L}^{2, \lambda}$. Ann. Mat. Pura e Appl. 69, 321-380 (1965)

[9] Campanato, S., Cannarsa, P.: Differentiability and partial Hölder continuity of the solutions of non - linear elliptic systems of order $2 m$ with quadratic growth. Annali della Scuola Normale Superiore di Pisa, Classe di Scienze, serie 4, tomo 8 2, 285-309 (1981)

[10] Chiarenza, F., Frasca, M., Longo, P.: Interior $W^{2, p}$ estimates for nondivergence elliptic equations with discontinuous coefficients. Ricerche di Matem. 40, 149-168 (1991)

[11] Chiarenza, F., Frasca, M., Longo, P.: $W^{2, p}$ solvability of the Dirichlet problem for nondivergence form elliptic equations with VMO coefficients. Trans. Am. Math. Soc. 336, 841-853 (1993)

[12] Coifman, R., Rochberg, R., Weiss, G.: Factorization theorems for Hardy spaces in several variables. Ann. Math. 103, 611-635 (1976)

[13] Dong, H., Kim, D.: Parabolic and elliptic systems in divergence form with variably partially BMO coefficients. SIAM J. Math. Anal. 43(3), 1075-1098 (2011)

[14] Farroni, F., Greco, L., Moscariello, G., Zecca, G.: Noncoercive quasilinear elliptic operators with singular lower order terms. Calc. Var. 60, 83 (2021)

[15] Giannetti, F., Greco, L., Moscariello, G.: Linear elliptic equations with lower-order terms. Differ. Int. Equ. 26, 623-638 (2013)

[16] Giannetti, F., Moscariello, G.: $W^{2,2}$ solvability of the Dirichlet problem for a class of elliptic equations with discontinuous coefficients. Rend. Lincei Mat. Appl. 29, 557-577 (2018) 
[17] Giaquinta, M.: Multiple integrals in the calculus of variations and nonlinear elliptic systems. In: Annals of Mathematics Studies, vol. 105. Princeton University Press, Princeton, NJ (1983)

[18] Giusti, E.: Direct Methods in the Calculus of Variations. World Scientific Publishing Co., Inc, River Edge (2003)

[19] Greco, L., Moscariello, G., Radice, T.: Nondivergence elliptic equations with unbounded coefficients. Discrete Contin. Dyn. Syst. B 11(1), 131-143 (2009)

[20] Hajlasz, P.: Sobolev spaces on an arbitrary metric space. Potential Anal. 5, 403-415 (1996)

[21] Iwaniec, T., Sbordone, C.: Weak minima of variational integrals. J. Reine Angew. Math. 454, 143-161 (1994)

[22] John, F., Nirenberg, L.: On functions of bounded mean oscillation. Commun. Pure Appl. Math. 14, 415-426 (1961)

[23] Kristensen, J., Mingione, G.: Sketches of regularity theory from the 20th century and the work of Jindrich Necas. Selected Works of Jindrich Necas. In: The Advances in Mathematical Fluid Mechanics. Springer Verlag (2013)

[24] Mingione, G.: Regularity of minima: an invitation to the dark side of the calculus of variations. Appl. Math. 51(4), 355-426 (2006)

[25] Miranda, C.: Alcune limitazioni integrali per le soluzioni delle equazioni lineari ellittiche di secondo ordine. Ann. Mat. Pura Appl. 49, 375-384 (1960)

[26] Morrey, C.B., Jr.: Second order elliptic systems of differential equations. Ann. Math. Stud. 33, 101-159 (1954)

[27] O'Neil, R.: Fractional Integration in Orlicz spaces I. Am. Math. Soc. 115, 300-328 (1965)

[28] Stroffolini, B.: Elliptic systems of PDE with BMO-coefficients. Potential Anal. 15, 285$299(2001)$

Gioconda Moscariello and Giulio Pascale

Dipartimento di Matematica e Applicazioni "R. Caccioppoli"

Universitá degli Studi di Napoli "Federico II"

via Cintia

80126 Naples

Italy

e-mail: gmoscari@unina.it

Giulio Pascale

e-mail: giulio.pascale@unina.it

Received: June 19, 2021.

Accepted: October 16, 2021. 